\title{
Jessen's functional, its properties and applications
}

\author{
Mario Krnić, Neda Lovričević and Josip Pečarić
}

\begin{abstract}
In this paper we consider Jessen's functional, defined by means of a positive isotonic linear functional, and investigate its properties. Derived results are then applied to weighted generalized power means, which yields extensions of some recent results, known from the literature. In particular, we obtain the whole series of refinements and converses of numerous classical inequalities such as the arithmetic-geometric mean inequality, Young's inequality and Hölder's inequality.
\end{abstract}

\section{Introduction}

Jensen's inequality is sometimes called the king of inequalities since it implies the whole series of other classical inequalities (e.g. those by Hölder, Minkowski, Beckenbach-Dresher and Young, the arithmetic-geometric mean inequality etc.). As we know, Jensen's inequality for convex functions is probably one of the most important inequalities which is extensively used in almost all areas of mathematics, especially in mathematical analysis and statistics. For a comprehensive inspection of the classical and recent results related to this inequality the reader is referred to [17] and [21].

Key Words: Inequalities, convex function, Jensen's inequality, Jessen's inequality, isotonic functional, Jessen's functional, superadditivity, subadditivity, monotonicity, arithmeticgeometric mean inequality, Young's inequality, Hölder's inequality.

2010 Mathematics Subject Classification: 26D15, 26A51.

Received: January, 2011.

Accepted: February, 2012 
In this paper we refer to the so called Jensen's functional, deduced from Jensen's inequality. Namely, Dragomir et al. (see [12]), investigated the properties of discrete Jensen's functional

$$
J_{n}(\Phi, \mathbf{x}, \mathbf{p})=\sum_{i=1}^{n} p_{i} \Phi\left(x_{i}\right)-P_{n} \Phi\left(\frac{\sum_{i=1}^{n} p_{i} x_{i}}{P_{n}}\right),
$$

where $\Phi: I \subset \mathbb{R} \rightarrow \mathbb{R}, \mathbf{x}=\left(x_{1}, x_{2}, \ldots, x_{n}\right) \in I^{n}, n \geq 2$, and $\mathbf{p}=$ $\left(p_{1}, p_{2}, \ldots, p_{n}\right)$ is the positive $n$-tuple of real numbers with $P_{n}=\sum_{i=1}^{n} p_{i}$. They obtained that, under the assumption that $\Phi$ is a convex function, such a functional is superadditive on the set of positive real $n$-tuples, that is

$$
J_{n}(\Phi, \mathbf{p}+\mathbf{q}, x) \geq J_{n}(\Phi, \mathbf{p}, x)+J_{n}(\Phi, \mathbf{q}, x) .
$$

Further, the above functional is also increasing in the same setting, that is,

$$
J_{n}(\Phi, \mathbf{p}, x) \geq J_{n}(\Phi, \mathbf{q}, x) \geq 0,
$$

where $\mathbf{p} \geq \mathbf{q}$ (i.e. $\left.p_{i} \geq q_{i}, i=1,2, \ldots, n\right)$. Monotonicity property of discrete Jensen's functional was proved few years before (see [17], p.717). The above mentioned properties provided refinements of numerous classical inequalities. For more details about such extensions see [12].

Very recently, Dragomir (see [13]) investigated boundedness of normalized Jensen's functional, that is the functional (1) satisfying $\sum_{i=1}^{n} p_{i}=1$. $\mathrm{He}$ obtained the following lower and upper bound for normalized functional:

$$
\max _{1 \leq i \leq n}\left\{\frac{p_{i}}{q_{i}}\right\} J_{n}(\Phi, \mathbf{x}, \mathbf{q}) \geq J_{n}(\Phi, \mathbf{x}, \mathbf{p}) \geq \min _{1 \leq i \leq n}\left\{\frac{p_{i}}{q_{i}}\right\} J_{n}(\Phi, \mathbf{x}, \mathbf{q}) \geq 0
$$

In relation (4), $\Phi: K \subset X \rightarrow X$ is convex function on convex subset $K$ of linear space $X, \mathbf{x}=\left(x_{1}, x_{2}, \ldots, x_{n}\right) \in K^{n}$, and $\mathbf{p}=\left(p_{1}, p_{2}, \ldots, p_{n}\right), \mathbf{q}=$ $\left(q_{1}, q_{2}, \ldots, q_{n}\right)$ are positive real $n$-tuples with $\sum_{i=1}^{n} p_{i}=\sum_{i=1}^{n} q_{i}=1$. Let's mention that an alternative proof of relation (4) was also given in [8].

It is well known that Jensen's inequality can be regarded in a more general manner, including positive linear functionals acting on linear class of real valued functions.

More precisely, let $E$ be nonempty set and $\mathcal{L}(E, \mathbb{R})$ linear class of realvalued functions $f: E \rightarrow \mathbb{R}$ satisfying following properties:

L1: $f, g \in \mathcal{L}(E, \mathbb{R}) \Rightarrow \alpha f+\beta g \in \mathcal{L}(E, \mathbb{R})$ for all $\alpha, \beta \in \mathbb{R}$

L2: $1 \in \mathcal{L}(E, \mathbb{R})$, that is, if $f(t)=1$ for all $t \in E$, then $f \in \mathcal{L}(E, \mathbb{R})$.

We also consider isotonic positive linear functionals $A: \mathcal{L}(E, \mathbb{R}) \rightarrow \mathbb{R}$. That is, we assume that 
A1: $A(\alpha f+\beta g)=\alpha A(f)+\beta A(g)$ for $f, g \in \mathcal{L}(E, \mathbb{R}), \alpha, \beta \in \mathbb{R}$ (linearity);

A2: $f \in \mathcal{L}(E, \mathbb{R}), f(t) \geq 0$ for all $t \in E \Rightarrow A(f) \geq 0$ (isotonicity).

Further, if

A3: $A(1)=1$

also holds, we say that $A$ is normalized isotonic positive linear functional or $A(f)$ is linear mean defined on $\mathcal{L}(E, \mathbb{R})$.

Common examples of such isotonic functionals $A$ are given by

$$
A(f)=\int_{E} f d \mu \quad \text { or } \quad A(f)=\sum_{k \in E} p_{k} f_{k}
$$

where $\mu$ is a positive measure on $E$ in the first case, and $E$ is subset of $\mathbb{N}=\{1,2, \ldots\}$ with all $p_{k}>0$ in the second case. Additionally, if $E$ is an interval $\langle a, b\rangle$, where $-\infty \leq a<b \leq \infty$, and

$$
\mathcal{L}(E, \mathbb{R})=\left\{f: E \rightarrow \mathbb{R} ; \alpha(f)=\lim _{x \rightarrow a^{+}} f(x), \beta(f)=\lim _{x \rightarrow b^{-}} f(x) \text { both exist }\right\},
$$

then $A(f)=\alpha(f)+\beta(f)$ or $A(f)=[\alpha(f)+\beta(f)] / 2$ or $A(f)=\alpha(f)$, etc., are also isotonic linear functionals.

Jessen's generalization of Jensen's inequality (see [21], p. 47-48), in view of positive isotonic functionals, claims that

$$
\Phi(A(f)) \leq A(\Phi(f))
$$

where $\Phi$ is continuous convex function on interval $I \subseteq \mathbb{R}, A$ is normalized isotonic positive linear functional, and $f \in \mathcal{L}(E, \mathbb{R})$ such that $\Phi(f) \in$ $\mathcal{L}(E, \mathbb{R})$. Jessen's inequality was extensively studied during the eighties and early nineties of the last century (see papers [9], [11], [15], [18], [19], [20], [23]).

Very recently, Culjak et al. (see [10]) generalized Jessen's relation (5). Namely, suppose $\Phi$ is continuous convex function on real interval, $p, q \in$ $\mathcal{L}(E, \mathbb{R})$ are non-negative functions, and let the non-negative constants $m$ and $M$ exist such that $p(t)-m q(t) \geq 0, M q(t)-p(t) \geq 0, A(p)-m A(q)>0$, $M A(q)-A(p)>0$. Then, the series of inequalities

$$
\begin{aligned}
M\left[A(q \Phi(f))-A(q) \Phi\left(\frac{A(q f)}{A(q)}\right)\right] & \geq A(p \Phi(f))-A(p) \Phi\left(\frac{A(p f)}{A(p)}\right) \\
& \left.\geq m\left[A(q \Phi(f))-A(q) \Phi\left(\frac{A(q f)}{A(q)}\right)\right] 6\right)
\end{aligned}
$$

hold for every $f \in \mathcal{L}(E, \mathbb{R})$ such that all expressions in (6) are well defined. 
Led by Jessen's variant of Jensen's inequality, in this paper we study the so called Jessen's functional which includes the described isotonic functional. We shall obtain that the mentioned properties of superadditivity and monotonicity hold in a more general manner. These properties can also be regarded as refinements and converses of numerous classical inequalities, what will be considered in the sequel. Results that will be deduced in the paper, generalize all the mentioned results in this Introduction.

The paper is organized in the following way: After this Introduction, in Section 2 we define Jessen's functional deduced from inequality (5), and analyze its properties depending on convexity of associated function. Further, in Section 3 we apply our general results to the weighted general and power means with respect to positive isotonic linear functional. In particular, we consider the obtained results in various settings what will bring us to the improvements of some earlier results, known from the literature. The last Section 4 is dedicated to inequalities of Hölder's type and their improvements.

The techniques that will be used in the proofs are mainly based on classical real analysis, especially on the well known Jensen's inequality.

\section{Definition and basic properties of generalized Jensen's functional}

In this section, by means of relation (5), we define Jessen's functional including positive isotonic functional. Before we define such functional, we have to establish some basic notation.

Let $\mathcal{F}(I, \mathbb{R})$ be the linear space of all real functions on interval $I \subseteq \mathbb{R}$, let $\mathcal{L}(E, \mathbb{R})$ be the linear class of real functions, defined on nonempty set $E$, satisfying properties $(\mathrm{L} 1)$ and $(\mathrm{L} 2)$, and let $\mathcal{L}_{0}^{+}(E, \mathbb{R}) \subset \mathcal{L}(E, \mathbb{R})$ be subset of non-negative functions in $\mathcal{L}(E, \mathbb{R})$. Further, let $\mathcal{J}(\mathcal{L}(E, \mathbb{R}), \mathbb{R})$ denotes the space of positive isotonic linear functionals on $\mathcal{L}(E, \mathbb{R})$, that is, we assume that such functionals satisfy properties (A1) and (A2).

As a generalization of Jensen's functional, with respect to isotonic functional, we define $J: \mathcal{F}(I, \mathbb{R}) \times \mathcal{L}(E, \mathbb{R}) \times \mathcal{L}_{0}^{+}(E, \mathbb{R}) \times \mathcal{J}(\mathcal{L}(E, \mathbb{R}), \mathbb{R}) \rightarrow \mathbb{R}$ as

$$
J(\Phi, f, p ; A)=A(p \Phi(f))-A(p) \Phi\left(\frac{A(p f)}{A(p)}\right) .
$$

Clearly, definition (7) is deduced from relation (5) and it also contains definition (1) of discrete Jensen's functional. We call (7) Jessen's functional.

Remark 1. In the above definition (7) we suppose $p f, p \Phi(f) \in \mathcal{L}(E, \mathbb{R})$. Then, it is easy to see that $\Phi\left(\frac{A(p f)}{A(p)}\right)$ is well defined provided that $A(p) \neq 0$. 
Namely, $A_{1}(f)=\frac{A(p f)}{A(p)} \in \mathcal{J}(\mathcal{L}(E, \mathbb{R}), \mathbb{R})$ is normalized isotonic functional, that is, $A_{1}(1)=1$. Now, suppose $I=[a, b]$. Clearly, $a \leq f(t) \leq b$, $\forall t \in E$. Since $b-f(t) \geq 0$, by using properties (A1), (A2), and (A3) we have $b-A_{1}(f)=A_{1}(b)-A_{1}(f)=A_{1}(b-f) \geq 0$, hence $A_{1}(f) \leq b$. Similarly, $a \leq A_{1}(f)$ wherefrom we conclude that $\frac{A(p f)}{A(p)}$ belongs to interval $I$.

Conditions similar to those in Remark 1 will usually be omitted, so Jessen's functional (7) will initially assumed to be well defined.

Remark 2. If $\Phi$ is continuous convex function on interval $I$, then Jessen's functional is non-negative, i.e.

$$
J(\Phi, f, p ; A) \geq 0 .
$$

It follows directly from Jessen's relation (5) applied on normalized isotonic functional $A_{1}(f)=\frac{A(p f)}{A(p)} \in \mathcal{J}(\mathcal{L}(E, \mathbb{R}), \mathbb{R})$. On the other hand, if $\Phi$ is continuous concave function, then the sign of inequality in (8) is reversed.

Now we are ready to state and prove our main result that describes basic properties of Jessen's functional.

Theorem 1. Suppose $\Phi: I \subset \mathbb{R} \rightarrow \mathbb{R}$ is continuous convex function. Let $f \in \mathcal{L}(E, \mathbb{R}), p, q \in \mathcal{L}_{0}^{+}(E, \mathbb{R}), A \in \mathcal{J}(\mathcal{L}(E, \mathbb{R}), \mathbb{R})$, such that Jessen's functional (7) is well defined. Then, functional ( 7$)$ possess the following properties:

(i) $J(\Phi, f, \cdot ; A)$ is superadditive on $\mathcal{L}_{0}^{+}(E, \mathbb{R})$, i.e.

$$
J(\Phi, f, p+q ; A) \geq J(\Phi, f, p ; A)+J(\Phi, f, q ; A) .
$$

(ii) If $p, q \in \mathcal{L}_{0}^{+}(E, \mathbb{R})$ with $p \geq q$, then

$$
J(\Phi, f, p ; A) \geq J(\Phi, f, q ; A) \geq 0,
$$

i.e. $J(\Phi, f, \cdot ; A)$ is increasing on $\mathcal{L}_{0}^{+}(E, \mathbb{R})$.

(iii) If $\Phi$ is continuous concave function, then the signs of inequality in (9) and (10) are reversed, i.e. $J(\Phi, f, \cdot ; A)$ is subadditive and decreasing on $\mathcal{L}_{0}^{+}(E, \mathbb{R})$.

Proof. (i) From definition (7) and by using the linearity of isotonic functional $A$, we have

$$
\begin{aligned}
& J \quad(\Phi, f, p+q ; A)=A((p+q) \Phi(f))-A(p+q) \Phi\left(\frac{A((p+q) f)}{A(p+q)}\right) \\
& =\quad A(p \Phi(f)+q \Phi(f))-(A(p)+A(q)) \Phi\left(\frac{A(p f+q f)}{A(p)+A(q)}\right) \\
& =A(p \Phi(f))+A(q \Phi(f))-(A(p)+A(q)) \Phi\left(\frac{A(p f)+A(q f)}{A(p)+A(q)}\right) .
\end{aligned}
$$


On the other hand, the convexity of function $\Phi$, together with classical Jensen's inequality, yields inequality

$$
\begin{aligned}
\Phi\left(\frac{A(p f)+A(q f)}{A(p)+A(q)}\right) & =\Phi\left(\frac{A(p)}{A(p)+A(q)} \cdot \frac{A(p f)}{A(p)}+\frac{A(q)}{A(p)+A(q)} \cdot \frac{A(q f)}{A(q)}\right) \\
& \leq \frac{A(p)}{A(p)+A(q)} \Phi\left(\frac{A(p f)}{A(p)}\right)+\frac{A(q)}{A(p)+A(q)} \Phi\left(\frac{A(q f)}{A(q)}\right),
\end{aligned}
$$

which can be rewritten in the following form:

$$
(A(p)+A(q)) \Phi\left(\frac{A(p f)+A(q f)}{A(p)+A(q)}\right) \leq A(p) \Phi\left(\frac{A(p f)}{A(p)}\right)+A(q) \Phi\left(\frac{A(q f)}{A(q)}\right) .
$$

Finally, by combining relation (11) and inequality (12) we get

$$
\begin{aligned}
J(\Phi, f, p+q ; A) & \geq A(p \Phi(f))+A(q \Phi(f))-A(p) \Phi\left(\frac{A(p f)}{A(p)}\right)- \\
& -A(q) \Phi\left(\frac{A(q f)}{A(q)}\right)=J(\Phi, f, p ; A)+J(\Phi, f, q ; A),
\end{aligned}
$$

that is, superadditivity of $J(\Phi, f, \cdot ; A)$ on $\mathcal{L}_{0}^{+}(E, \mathbb{R})$.

(ii) Monotonicity follows easily from superadditivity. Since $p \geq q \geq 0$, we can represent $p \in \mathcal{L}_{0}^{+}(E, \mathbb{R})$ as the sum of two functions in $\mathcal{L}_{0}^{+}(E, \mathbb{R})$, namely $p=(p-q)+q$. Now, from relation (9) we get

$$
J(\Phi, f, p ; A)=J(\Phi, f, p-q+q ; A) \geq J(\Phi, f, p-q ; A)+J(\Phi, f, q ; A) .
$$

Finally, since $J(\Phi, f, p-q ; A) \geq 0$, it follows that $J(\Phi, f, p ; A) \geq J(\Phi, f, q ; A)$, which completes the proof.

(iii) The case of concave function is treated in the same way as in (i) and (ii), taking into consideration that the sign of Jensen's inequality is reversed and that Jessen's functional is non-positive in that case.

The properties contained in Theorem 1 play meaningful role in numerous applications of Jessen's inequality. As the first consequence of Theorem 1, we consider monotonicity property of Jessen's functional which includes the function that attains minimum and maximum value on its domain. That result is contained in the following statement.

Corollary 1. Let $\Phi$ be continuous convex function on real interval, let $f \in$ $\mathcal{L}(E, \mathbb{R})$, and let $A \in \mathcal{J}(\mathcal{L}(E, \mathbb{R}), \mathbb{R})$. Suppose $p \in \mathcal{L}_{0}^{+}(E, \mathbb{R})$ attains minimum and maximum value on the set $E$. If the functional $(7)$ is well defined, then the following series of inequalities hold

$$
\left[\max _{x \in E} p(x)\right] j(\Phi, f ; A) \geq J(\Phi, f, p ; A) \geq\left[\min _{x \in E} p(x)\right] j(\Phi, f ; A),
$$


where

$$
j(\Phi, f ; A)=A(\Phi(f))-A(1) \Phi\left(\frac{A(f)}{A(1)}\right) .
$$

Further, if $\Phi$ is continuous concave function, then the signs of inequality in (13) are reversed.

Proof. The result follows easily from property (10) of Jessen's functional. Namely, since $p \in \mathcal{L}_{0}^{+}(E, \mathbb{R})$ attains minimum and maximum value on its domain $E$, then

$$
\max _{x \in E} p(x) \geq p(x) \geq \min _{x \in E} p(x),
$$

so we can consider two constant functions

$$
\bar{p}(x)=\max _{x \in E} p(x) \quad \text { and } \quad \underline{p}(x)=\min _{x \in E} p(x) .
$$

Now, double application of property (10) yields required result since

$J(\Phi, f, \bar{p} ; A)=\left[\max _{x \in E} p(x)\right] j(\Phi, f ; A)$ and $J(\Phi, f, \underline{p} ; A)=\left[\min _{x \in E} p(x)\right] j(\Phi, f ; A)$.

The properties described in Theorem 1 and Corollary 1 generalize all the results presented in the Introduction, what will clearly be explained in next few remarks. Additionally, obtained results provide new opportunities for applications of Jessen's inequality, what will be discussed in the sequel.

Remark 3. Our main result, i.e. Theorem 1 is the generalization of relation (6) from the Introduction. More precisely, suppose that the functions $p, q \in \mathcal{L}_{0}^{+}(E, \mathbb{R})$ are chosen in such a way that there exist positive real constants $m$ and $M$ such that relation $M q(x) \geq p(x) \geq m q(x)$ holds for all $x \in E$. Then, double application of property (10) yields (6) since $J(\Phi, f, m q ; A)=m J(\Phi, f, q ; A)$ and $J(\Phi, f, M q ; A)=M J(\Phi, f, q ; A)$.

Remark 4. Let's consider the discrete case of Theorem 1 or rather Corollary 1. We suppose $E=\{1,2, \ldots, n\}$ and $\mathcal{L}(E, \mathbb{R})$ is the class of real $n$-tuples. If we consider the discrete functional $A \in \mathcal{J}(\mathcal{L}(E, \mathbb{R}), \mathbb{R})$ defined by $A(\mathbf{x})=$ $\sum_{i=1}^{n} x_{i}$, where $\mathbf{x}=\left(x_{1}, x_{2}, \ldots, x_{n}\right)$, then the functional (7) becomes discrete functional (1) from paper [12]. Additionally, if $\mathbf{p}=\left(p_{1}, p_{2}, \ldots, p_{n}\right)$ with $\sum_{i=1}^{n} p_{i}=1$, we get normalized Jensen's functional from relation (4) (see paper [13]). Of course, our Theorem 1 is further generalization of relation (4) from the Introduction. Namely, if $\mathbf{p}=\left(p_{1}, p_{2}, \ldots, p_{n}\right)$ and $\mathbf{q}=\left(q_{1}, q_{2}, \ldots, q_{n}\right)$ 
are two positive $n$-tuples satisfying $\sum_{i=1}^{n} p_{i}=1$ and $\sum_{i=1}^{n} q_{i}=1$, then, if we denote

$$
m=\min _{1 \leq i \leq n}\left\{\frac{p_{i}}{q_{i}}\right\} \quad \text { and } \quad M=\max _{1 \leq i \leq n}\left\{\frac{p_{i}}{q_{i}}\right\},
$$

the relation $M q_{i} \geq p_{i} \geq m q_{i}$ holds for all $i=1,2, \ldots, n$, that is, we are in conditions of Remark 3 .

Remark 5. Let's rewrite relation (13) from Corollary 1 in a discrete form. Namely, under the same notations as in the previous remark, relation (13) takes form

$$
\max _{1 \leq i \leq n}\left\{p_{i}\right\} S_{\Phi}(\mathbf{x}) \geq J_{n}(\Phi, \mathbf{x}, \mathbf{p}) \geq \min _{1 \leq i \leq n}\left\{p_{i}\right\} S_{\Phi}(\mathbf{x})
$$

where the functional $J_{n}(\Phi, \mathbf{x}, \mathbf{p})$ is defined by (1) and $S_{\Phi}(\mathbf{x})=\sum_{i=1}^{n} \Phi\left(x_{i}\right)-$ $n \Phi\left(\frac{\sum_{i=1}^{n} x_{i}}{n}\right)$. Relation (15) was proved in [24] only for $n=2$ in the case of normalized functional. The mentioned relation for $n=2$ was also used in applications of Jensen's inequality. For example, in [14], the authors use (15), for $n=2$, in obtaining some global upper bounds for Jensen's inequality.

We conclude this section with remark about integral form of Jessen's functional.

Remark 6. Suppose $E \subseteq \mathbb{R}$ and let $\mathcal{L}(E, \mathbb{R})$ be linear class of measurable functions with respect to positive measure $\mu$. If $A(f)=\int_{E} f d \mu$, then the integral representation of functional (7) reads

$$
\int_{E} p(x) \Phi(f(x)) d \mu(x)-\left(\int_{E} p(x) d \mu(x)\right) \Phi\left(\frac{\int_{E} p(x) f(x) d \mu(x)}{\int_{E} p(x) d \mu(x)}\right) .
$$

\section{Applications to weighted generalized and power means}

In this section we apply our basic results from the previous section to weighted generalized and power means with respect to isotonic functional $A \in \mathcal{J}(\mathcal{L}(E, \mathbb{R})$, $\mathbb{R})$. In such a manner, we obtain both refinements and converses of numerous classical inequalities, what will briefly be discussed in the sequel.

Recall, weighted generalized mean with respect to isotonic linear functional $A \in \mathcal{J}(\mathcal{L}(E, \mathbb{R}), \mathbb{R})$ and continuous and strictly monotone function $\chi \in \mathcal{F}(I, \mathbb{R})$, 
is defined as

$$
M_{\chi}(f, p ; A)=\chi^{-1}\left(\frac{A(p \chi(f))}{A(p)}\right), f \in \mathcal{L}(E, \mathbb{R}), p \in \mathcal{L}_{0}^{+}(E, \mathbb{R}) .
$$

Of course, we assume that (16) is well defined, that is, $A(p) \neq 0$ and $p \chi(f) \in$ $\mathcal{L}(E, \mathbb{R})$. Similarly as in the previous section, such conditions will usually be omitted, so the weighted generalized mean (16) will initially assumed to be well defined.

Theorem 2. Let $\chi, \psi \in \mathcal{F}(I, \mathbb{R})$ be continuous and strictly monotone functions such that the function $\chi \circ \psi^{-1}$ is convex. Suppose $f \in \mathcal{L}(E, \mathbb{R}), p, q \in$ $\mathcal{L}_{0}^{+}(E, \mathbb{R}), A \in \mathcal{J}(\mathcal{L}(E, \mathbb{R}), \mathbb{R})$ are such that the functional

$$
A(p)\left[\chi\left(M_{\chi}(f, p ; A)\right)-\chi\left(M_{\psi}(f, p ; A)\right)\right]
$$

is well defined. Then, functional (17) satisfies the following properties:

(i) $A(\cdot)\left[\chi\left(M_{\chi}(f, \cdot ; A)\right)-\chi\left(M_{\psi}(f, \cdot ; A)\right)\right]$ is superadditive on $\mathcal{L}_{0}^{+}(E, \mathbb{R})$, i.e.

$$
\begin{aligned}
A(p+q) & {\left[\chi\left(M_{\chi}(f, p+q ; A)\right)-\chi\left(M_{\psi}(f, p+q ; A)\right)\right] } \\
\geq & A(p)\left[\chi\left(M_{\chi}(f, p ; A)\right)-\chi\left(M_{\psi}(f, p ; A)\right)\right] \\
& +A(q)\left[\chi\left(M_{\chi}(f, q ; A)\right)-\chi\left(M_{\psi}(f, q ; A)\right)\right] .
\end{aligned}
$$

(ii) If $p, q \in \mathcal{L}_{0}^{+}(E, \mathbb{R})$ with $p \geq q$, then

$$
\begin{aligned}
A(p) & {\left[\chi\left(M_{\chi}(f, p ; A)\right)-\chi\left(M_{\psi}(f, p ; A)\right)\right] } \\
& \geq A(q)\left[\chi\left(M_{\chi}(f, q ; A)\right)-\chi\left(M_{\psi}(f, q ; A)\right)\right],
\end{aligned}
$$

that is, $A(\cdot)\left[\chi\left(M_{\chi}(f, \cdot ; A)\right)-\chi\left(M_{\psi}(f, \cdot ; A)\right)\right]$ is increasing on $\mathcal{L}_{0}^{+}(E, \mathbb{R})$.

(iii) If $\chi \circ \psi^{-1}$ is concave function, then the signs of inequality in (18) and (19) are reversed, that is, $A(\cdot)\left[\chi\left(M_{\chi}(f, \cdot ; A)\right)-\chi\left(M_{\psi}(f, \cdot ; A)\right)\right]$ is subadditive and decreasing on $\mathcal{L}_{0}^{+}(E, \mathbb{R})$.

Proof. We consider Jessen's functional (7) where the convex function $\Phi$ is replaced with $\chi \circ \psi^{-1}$ and $f \in \mathcal{L}(E, \mathbb{R})$ with $\psi(f) \in \mathcal{L}(E, \mathbb{R})$. Thus, having in mind definition (16), functional (7) can be rewritten in the form

$$
\begin{aligned}
J\left(\chi \circ \psi^{-1}, \psi(f), p ; A\right) & =A\left(p \cdot\left(\chi \circ \psi^{-1}(\psi(f))\right)\right)-A(p) \chi\left(\psi^{-1}\left(\frac{A(p \psi(f))}{A(p)}\right)\right) \\
& =A(p \chi(f))-A(p) \chi\left(M_{\psi}(f, q ; A)\right) \\
& =A(p) \chi\left(M_{\chi}(f, p ; A)\right)-A(p) \chi\left(M_{\psi}(f, q ; A)\right) \\
& =A(p)\left[\chi\left(M_{\chi}(f, p ; A)\right)-\chi\left(M_{\psi}(f, p ; A)\right)\right] .
\end{aligned}
$$


Now, the properties (i), (ii), and (iii) follow immediately from Theorem 1.

Similarly as in Section 2, we can bound functional (17) with the minimum and maximum value of function $p \in \mathcal{L}_{0}^{+}(E, \mathbb{R})$ if they exist.

Corollary 2. Let $\chi, \psi \in \mathcal{F}(I, \mathbb{R})$ be continuous and strictly monotone functions such that the function $\chi \circ \psi^{-1}$ is convex. Suppose $f \in \mathcal{L}(E, \mathbb{R}), p \in$ $\mathcal{L}_{0}^{+}(E, \mathbb{R}), A \in \mathcal{J}(\mathcal{L}(E, \mathbb{R}), \mathbb{R})$ are such that the functional $(17)$ is well defined. If $p \in \mathcal{L}_{0}^{+}(E, \mathbb{R})$ attains minimum and maximum value on its domain $E$, then

$$
\begin{aligned}
& {\left[\max _{x \in E} p(x)\right] A(1)\left[\chi\left(m_{\chi}(f ; A)\right)-\chi\left(m_{\psi}(f ; A)\right)\right]} \\
& \geq A(p)\left[\chi\left(M_{\chi}(f, p ; A)\right)-\chi\left(M_{\psi}(f, p ; A)\right)\right] \\
& \geq\left[\min _{x \in E} p(x)\right] A(1)\left[\chi\left(m_{\chi}(f ; A)\right)-\chi\left(m_{\psi}(f ; A)\right)\right],
\end{aligned}
$$

where

$$
m_{\eta}(f ; A)=\eta^{-1}\left(\frac{A(\eta(f))}{A(1)}\right), \quad \eta=\chi, \psi .
$$

Additionally, if $\chi \circ \psi^{-1}$ is concave function, then the signs of inequality in (20) are reversed.

Proof. Follows directly from property (19), following the same lines as in the proof of Corollary 1.

The first consequence of Theorem 2 refers to generalized power means $M^{[r]}(f, p ; A), r \in \mathbb{R}$, equipped with isotonic functional $A \in \mathcal{J}(\mathcal{L}(E, \mathbb{R}), \mathbb{R})$. We have

$$
M^{[r]}(f, p ; A)=\left\{\begin{array}{ll}
\left(\frac{A\left(p f^{r}\right)}{A(p)}\right)^{\frac{1}{r}}, & r \neq 0 \\
\exp \left(\frac{A(p \ln (f))}{A(p)}\right), & r=0
\end{array},\right.
$$

where $f, p \in \mathcal{L}_{0}^{+}(E, \mathbb{R})$, and $f(x)>0$ for all $x \in E$. We assume that the above expression is well defined, that is, $p f^{r} \in \mathcal{L}_{0}^{+}(E, \mathbb{R}), p \ln (f) \in \mathcal{L}(E, \mathbb{R})$, and $A(p) \neq 0$. Such conditions will be omitted below.

Corollary 3. Let $s \neq 0$ and $r$ be real numbers, let $f, p, q \in \mathcal{L}_{0}^{+}(E, \mathbb{R}), f(x)>$ $0, \forall x \in E$, and let $A \in \mathcal{J}(\mathcal{L}(E, \mathbb{R}), \mathbb{R})$. If the functional

$$
A(p)\left\{\left[M^{[s]}(f, p ; A)\right]^{s}-\left[M^{[r]}(f, p ; A)\right]^{s}\right\}
$$


is well defined, then it has the following properties:

(i) If $s>0, s>r$ or $s<0, s<r$ or $r=0$, then

$$
\begin{aligned}
A(p+q) & \left\{\left[M^{[s]}(f, p+q ; A)\right]^{s}-\left[M^{[r]}(f, p+q ; A)\right]^{s}\right\} \\
\geq & A(p)\left\{\left[M^{[s]}(f, p ; A)\right]^{s}-\left[M^{[r]}(f, p ; A)\right]^{s}\right\} \\
& +A(q)\left\{\left[M^{[s]}(f, q ; A)\right]^{s}-\left[M^{[r]}(f, q ; A)\right]^{s}\right\}
\end{aligned}
$$

i.e. $A(\cdot)\left\{\left[M^{[s]}(f, \cdot ; A)\right]^{s}-\left[M^{[r]}(f, \cdot ; A)\right]^{s}\right\}$ is superadditive on $\mathcal{L}_{0}^{+}(E, \mathbb{R})$.

(ii) If $s>0, s>r$ or $s<0, s<r$ or $r=0$, then for $p, q \in \mathcal{L}_{0}^{+}(E, \mathbb{R})$ with $p \geq q$, holds inequality

$$
\begin{aligned}
A(p)\{ & {\left.\left[M^{[s]}(f, p ; A)\right]^{s}-\left[M^{[r]}(f, p ; A)\right]^{s}\right\} } \\
& \geq A(q)\left\{\left[M^{[s]}(f, q ; A)\right]^{s}-\left[M^{[r]}(f, q ; A)\right]^{s}\right\}
\end{aligned}
$$

i.e. functional $A(\cdot)\left\{\left[M^{[s]}(f, \cdot ; A)\right]^{s}-\left[M^{[r]}(f, \cdot ; A)\right]^{s}\right\}$ is increasing on $\mathcal{L}_{0}^{+}(E$, $\mathbb{R})$.

(iii) If $s>0, s<r$ or $s<0, s>r$, then the signs of inequality in (24) and (25) are reversed, that is, $A(\cdot)\left\{\left[M^{[s]}(f, \cdot ; A)\right]^{s}-\left[M^{[r]}(f, \cdot ; A)\right]^{s}\right\}$ is subadditive and decreasing on $\mathcal{L}_{0}^{+}(E, \mathbb{R})$.

Proof. The proof is direct use of Theorem 2. We have to consider two cases depending on whether $r \neq 0$ or $r=0$.

If $r \neq 0$, we define $\chi(x)=x^{s}$ and $\psi(x)=x^{r}$. Then, $\chi \circ \psi^{-1}(x)=x^{\frac{s}{r}}$ and $\left(\chi \circ \psi^{-1}\right)^{\prime \prime}(x)=\frac{s(s-r)}{r^{2}} x^{\frac{s}{r}-2}$. Thus, $\chi \circ \psi^{-1}$ is convex if $s>0, s>r$ or $s<0, s<r$. On the other hand, $\chi \circ \psi^{-1}$ is concave if $s>0, s<r$ or $s<0, s>r$.

If $r=0$, we put $\chi(x)=x^{s}$ and $\psi(x)=\ln x$. Then, $\chi \circ \psi^{-1}(x)=e^{s x}$ is convex under assumption $s \neq 0$.

Now, the result follows immediately from Theorem 2 .

In addition, Corollary 2, applied on generalized power means, yields the following result.

Corollary 4. Let $s$ and $r$ be real numbers such that $s>0, s>r$ or $s<$ $0, s<r$ or $r=0, s \neq 0$. Suppose $f, p \in \mathcal{L}_{0}^{+}(E, \mathbb{R}), f(x)>0, \forall x \in E, A \in$ $\mathcal{J}(\mathcal{L}(E, \mathbb{R}), \mathbb{R})$ are such that the functional $(23)$ is well defined. If $p \in \mathcal{L}_{0}^{+}(E, \mathbb{R})$ 
attains minimum and maximum value on its domain $E$, then

$$
\begin{gathered}
{\left[\max _{x \in E} p(x)\right] A(1)\left\{\left[m^{[s]}(f ; A)\right]^{s}-\left[m^{[r]}(f ; A)\right]^{s}\right\}} \\
\geq A(p)\left\{\left[M^{[s]}(f, p ; A)\right]^{s}-\left[M^{[r]}(f, p ; A)\right]^{s}\right\} \\
\geq\left[\min _{x \in E} p(x)\right] A(1)\left\{\left[m^{[s]}(f ; A)\right]^{s}-\left[m^{[r]}(f ; A)\right]^{s}\right\}
\end{gathered}
$$

where

$$
m^{[t]}(f ; A)=\left\{\begin{array}{ll}
\left(\frac{A\left(f^{r}\right)}{A(1)}\right)^{\frac{1}{t}}, & t \neq 0 \\
\exp \left(\frac{A(\ln (f))}{A(1)}\right), & t=0
\end{array}, \quad t=r, s .\right.
$$

Further, if $s>0, s<r$ or $s<0, s>r$, then the signs of inequality in (26) are reversed.

Proof. Follows immediately from property (25) or Corollary 2.

Relations (20) and (26) can be regarded as both refinements and converses of weighted generalized and power means. Clearly, in both series of inequalities, one inequality provides refinement, while the other one yields converse of appropriate mean inequality. The following remark describes such characteristics on the example of classical arithmetic-geometric mean inequality.

Remark 7. For the sake of simplicity, we consider discrete variant of relation (26). Equally as in Remark 4, we suppose $E=\{1,2, \ldots, n\}, n \in \mathbb{N}$, and $\mathcal{L}(E, \mathbb{R})$ is a class of real $n$-tuples. We consider discrete functional $A \in$ $\mathcal{J}(\mathcal{L}(E, \mathbb{R}), \mathbb{R})$ defined by $A(\mathbf{x})=\sum_{i=1}^{n} x_{i}$, where $\mathbf{x}=\left(x_{1}, x_{2}, \ldots, x_{n}\right)$. Clearly, $A(\mathbf{1})=\sum_{i=1}^{n} 1=n$.

Then, if we put $s=1$ and $r=0$, series of inequalities (26) can be rewritten as

$$
\begin{gathered}
n \max _{1 \leq i \leq n}\left\{p_{i}\right\}\left[A_{n}(\mathbf{x})-G_{n}(\mathbf{x})\right] \geq P_{n}\left[M_{1}(\mathbf{x}, \mathbf{p})-M_{0}(\mathbf{x}, \mathbf{p})\right] \\
\geq n \min _{1 \leq i \leq n}\left\{p_{i}\right\}\left[A_{n}(\mathbf{x})-G_{n}(\mathbf{x})\right] \geq 0
\end{gathered}
$$

where $P_{n}=\sum_{i=1}^{n} p_{i}$,

$$
M_{r}(\mathbf{x}, \mathbf{p})=\left\{\begin{array}{cc}
\left(\frac{\sum_{i=1}^{n} p_{i} x_{i}^{r}}{P_{n}}\right)^{\frac{1}{r}}, & r \neq 0 \\
\left(\prod_{i=1}^{n} x_{i}^{p_{i}}\right)^{\frac{1}{P_{n}}}, & r=0
\end{array},\right.
$$




$$
A_{n}(\mathbf{x})=\frac{\sum_{i=1}^{n} x_{i}}{n}, \quad \text { and } \quad G_{n}(\mathbf{x})=\left(\prod_{i=1}^{n} x_{i}\right)^{\frac{1}{n}} .
$$

Obviously, the first sign of inequality in (28), from the left, provides converse of arithmetic-geometric mean inequality $\left(M_{1}(\mathbf{x}, \mathbf{p})\right.$ and $\left.M_{0}(\mathbf{x}, \mathbf{p})\right)$, while the second one yields refinement of observed inequality. We also say that (28) yields converse and refinement of arithmetic-geometric mean inequality in difference form. Let's mention that some variants of inequalities in (28) were recently studied in paper [6] of Aldaz. See also papers [1], [2], [3], [4], and [5].

Note that corollaries 3 and 4 do not cover the case when $s=0$ and $r \neq 0$. This case should be considered separately.

Corollary 5. Let $r \neq 0$ be real number, let $f, p, q \in \mathcal{L}_{0}^{+}(E, \mathbb{R}), f(x)>0$, $\forall x \in E$, and let $A \in \mathcal{J}(\mathcal{L}(E, \mathbb{R}), \mathbb{R})$. If the functional

$$
A(p)\left\{\frac{A(p \ln f)}{A(p)}-\ln \left[M^{[r]}(f, p ; A)\right]\right\}
$$

is well defined, then it possess the following properties:

(i) If $r<0$ then

$$
\begin{aligned}
A(p+q) & \left\{\frac{A((p+q) \ln f)}{A(p+q)}-\ln \left[M^{[r]}(f, p+q ; A)\right]\right\} \\
\geq & A(p)\left\{\frac{A(p \ln f)}{A(p)}-\ln \left[M^{[r]}(f, p ; A)\right]\right\} \\
& +A(q)\left\{\frac{A(q \ln f)}{A(q)}-\ln \left[M^{[r]}(f, q ; A)\right]\right\},
\end{aligned}
$$

i.e. $A(\cdot)\left\{\frac{A(\cdot \ln f)}{A(\cdot)}-\ln \left[M^{[r]}(f, \cdot ; A)\right]\right\}$ is superadditive on $\mathcal{L}_{0}^{+}(E, \mathbb{R})$.

(ii) If $r<0$ then for $p, q \in \mathcal{L}_{0}^{+}(E, \mathbb{R})$ with $p \geq q$, holds inequality

$$
\begin{aligned}
A(p) & \left\{\frac{A(p \ln f)}{A(p)}-\ln \left[M^{[r]}(f, p ; A)\right]\right\} \\
& \geq A(q)\left\{\frac{A(q \ln f)}{A(q)}-\ln \left[M^{[r]}(f, q ; A)\right]\right\},
\end{aligned}
$$

i.e. $A(\cdot)\left\{\frac{A(\cdot \ln f)}{A(\cdot)}-\ln \left[M^{[r]}(f, \cdot ; A)\right]\right\}$ is increasing on $\mathcal{L}_{0}^{+}(E, \mathbb{R})$.

(iii) If $r>0$ then the signs of inequality in (32) and (33) are reversed, that is, $A(\cdot)\left\{\frac{A(\cdot \ln f)}{A(\cdot)}-\ln \left[M^{[r]}(f, \cdot ; A)\right]\right\}$ is subadditive and decreasing on $\mathcal{L}_{0}^{+}(E, \mathbb{R})$. 
Proof. The proof is direct consequence of Theorem 2. We define $\chi(x)=\ln x$ and $\psi(x)=x^{r}$. Then, the function $\chi \circ \psi^{-1}(x)=\frac{1}{r} \ln x$ is convex if $r<0$ and concave if $r>0$. That completes the proof.

The analogue of Corollary 4 , that covers the case when $s=0$ and $r \neq 0$, is contained in the following result.

Corollary 6. Let $r<0$ be real number, let $f, p \in \mathcal{L}_{0}^{+}(E, \mathbb{R}), f(x)>0$, $\forall x \in E$, and let $A \in \mathcal{J}(\mathcal{L}(E, \mathbb{R}), \mathbb{R})$. Assume that functional (31) is well defined. If $p \in \mathcal{L}_{0}^{+}(E, \mathbb{R})$ attains minimum and maximum value on its domain $E$, then

$$
\begin{aligned}
{\left[\max _{x \in E} p(x)\right] A(1)\left\{\frac{A(\ln f)}{A(1)}-\ln \left[m^{[r]}(f ; A)\right]\right\} } \\
\geq A(p)\left\{\frac{A(p \ln f)}{A(p)}-\ln \left[M^{[r]}(f, p ; A)\right]\right\} \\
\geq\left[\min _{x \in E} p(x)\right] A(1)\left\{\frac{A(\ln f)}{A(1)}-\ln \left[m^{[r]}(f ; A)\right]\right\},
\end{aligned}
$$

where $m^{[r]}(f ; A)$ is defined by $(27)$. On the other hand, if $r>0$ then the signs of inequality in (34) are reversed.

Proof. Follows directly from property (33) or Corollary 2.

Corollary 6 is very interesting since it provides yet another set of refinements and converses of mean inequalities, but in so called quotient form. We shall clearly explain that facts on the example of arithmetic-geometric mean inequality, which is the content of the following remark.

Remark 8. Similarly as in Remark 7, we consider discrete variant of relation (34). Then, by using the same notation as in Remark 7, the term $A(p \ln f) / A(p)$ takes the form

$$
\frac{\sum_{i=1}^{n} p_{i} \ln x_{i}}{\sum_{i=1}^{n} p_{i}}=\ln \left(\prod_{i=1}^{n} x_{i}^{p_{i}}\right)^{\frac{1}{P_{n}}}=\ln M_{0}(\mathbf{x}, \mathbf{p})
$$

what is logarithm of geometric mean. Of course, $r=1$ yields arithmetic mean, so we use relation (34) with reversed signs of inequality.

Thus, after elimination of logarithm function, reversed series of inequalities in $(34)$ reads 


$$
\left[\frac{A_{n}(\mathbf{x})}{G_{n}(\mathbf{x})}\right]^{n \max _{1 \leq i \leq n}\left\{p_{i}\right\}} \geq\left[\frac{M_{1}(\mathbf{x}, \mathbf{q})}{M_{0}(\mathbf{x}, \mathbf{q})}\right]^{P_{n}} \geq\left[\frac{A_{n}(\mathbf{x})}{G_{n}(\mathbf{x})}\right]^{n \min _{1 \leq i \leq n}\left\{p_{i}\right\}} \geq 1
$$

where $P_{n}, M_{0}(\mathbf{x}, \mathbf{p}), M_{1}(\mathbf{x}, \mathbf{p}), A_{n}(\mathbf{x}), G_{n}(\mathbf{x})$ are defined in Remark 7 . Of course, previous set of inequalities can be regarded as both refinement and converse of the classical arithmetic geometric-mean inequality in quotient form.

Since the Young's inequality is closely related with arithmetic-geometric mean inequality, relations (28) and (35) also yield refinements and converses of Young's inequality. We describe that connection in detail.

Remark 9. Young's inequality is another variant of arithmetic-geometric mean inequality, so relations (28) and (35) provide refinements and converses of Young's inequality. For that sake, if $\mathbf{x}=\left(x_{1}, x_{2}, \ldots, x_{n}\right)$ and $\mathbf{p}=\left(p_{1}, p_{2}, \ldots, p_{n}\right)$, we denote

$$
\mathbf{x}^{\mathbf{p}}=\left(x_{1}^{p_{1}}, x_{2}^{p_{2}}, \ldots, x_{n}^{p_{n}}\right) \quad \text { and } \quad \mathbf{p}^{-1}=\left(\frac{1}{p_{1}}, \frac{1}{p_{2}}, \ldots, \frac{1}{p_{n}}\right) .
$$

Now, let $\mathbf{x}=\left(x_{1}, x_{2}, \ldots, x_{n}\right)$ and $\mathbf{p}=\left(p_{1}, p_{2}, \ldots, p_{n}\right)$ be positive $n$-tuples such that $\sum_{i=1}^{n} \frac{1}{p_{i}}=1$. Then, series of inequalities (28) and (35) can be rewritten in the form

$$
\left[\frac{A_{n}\left(\mathbf{x}^{\mathbf{p}}\right)}{G_{n}\left(\mathbf{x}^{\mathbf{p}}\right)}\right]^{n \max _{1 \leq i \leq n}\left\{\frac{1}{p_{i}}\right\}} \geq \frac{M_{1}\left(\mathbf{x}^{\mathbf{p}}, \mathbf{p}^{-1}\right)}{M_{0}\left(\mathbf{x}^{\mathbf{p}}, \mathbf{p}^{-1}\right)} \geq\left[\frac{A_{n}\left(\mathbf{x}^{\mathbf{p}}\right)}{G_{n}\left(\mathbf{x}^{\mathbf{p}}\right)}\right]^{n \min _{1 \leq i \leq n}\left\{\frac{1}{p_{i}}\right\}}
$$

and

$$
\begin{gathered}
n \max _{1 \leq i \leq n}\left\{\frac{1}{p_{i}}\right\}\left[A_{n}\left(\mathbf{x}^{\mathbf{p}}\right)-G_{n}\left(\mathbf{x}^{\mathbf{p}}\right)\right] \geq M_{1}\left(\mathbf{x}^{\mathbf{p}}, \mathbf{p}^{-1}\right)-M_{0}\left(\mathbf{x}^{\mathbf{p}}, \mathbf{p}^{-1}\right) \\
\geq n \min _{1 \leq i \leq n}\left\{\frac{1}{p_{i}}\right\}\left[A_{n}\left(\mathbf{x}^{\mathbf{p}}\right)-G_{n}\left(\mathbf{x}^{\mathbf{p}}\right)\right]
\end{gathered}
$$

where $P_{n}, M_{0}(\mathbf{x}, \mathbf{p}), M_{1}(\mathbf{x}, \mathbf{p}), A_{n}(\mathbf{x}), G_{n}(\mathbf{x})$ are defined in Remark 7. Clearly, relations (36) and (37) represent refinements and converses of Young's inequality in quotient and difference form.

Finally, let's take a look at relation (37) in the case $n=2$. Then, if we denote $1 / p_{1}=1-\nu, 1 / p_{2}=\nu, \nu \in\langle 0,1\rangle, x_{1}^{p_{1}}=a, x_{2}^{p_{2}}=b$, relation $(37)$ can be rewritten in the form

$$
\begin{aligned}
\max \{\nu, 1-\nu\}(\sqrt{a}-\sqrt{b})^{2} & \geq(1-\nu) a+\nu b-a^{1-\nu} b^{\nu} \\
& \geq \min \{\nu, 1-\nu\}(\sqrt{a}-\sqrt{b})^{2} .
\end{aligned}
$$


The second inequality in (38), that is refinement of Young's inequality for $n=2$, was very recently proved in [16]. So our relation (37) is a further generalization of the second inequality in (38), obtained in paper [16].

Finally, let's mention that, according to Remark 3, all the results from this section are generalizations of appropriate results from paper [10].

\section{Applications to Hölder's inequality}

This section is devoted to one of the most important inequalities in mathematical analysis, that is Hölder's inequality. In view of isotonic functional $A \in \mathcal{J}(\mathcal{L}(E, \mathbb{R}), \mathbb{R})$, Hölder's inequality claims that

$$
A\left(\prod_{i=1}^{n} f_{i}^{\frac{1}{p_{i}}}\right) \leq \prod_{i=1}^{n} A^{\frac{1}{p_{i}}}\left(f_{i}\right)
$$

where $p_{i}, i=1,2, \ldots, n$ are conjugate exponents, that is $\sum_{i=1}^{n} 1 / p_{i}=1, p_{i}>$ $1, i=1,2, \ldots, n$, and provided that $f_{1}, f_{2}, \ldots, f_{n}, \prod_{i=1}^{n} f_{i}{ }^{1 / p_{i}} \in \mathcal{L}_{0}^{+}(E, \mathbb{R})$.

It is well known from the literature (see [17] and [21]) that Hölder's inequality can easily be obtained from Young's inequality. On the other hand, in Remark 9 we have obtained refinements and converses of Young's inequality. Therefore, it is natural to expect that relations (36) and (37) also provide refinements and converses of Hölder's inequality.

The first in a series of results refers to relation (37), that is refinement and converse of Hölder's inequality in difference form.

Theorem 3. Let $p_{i}>1, i=1,2, \ldots, n$, be conjugate exponents, let $f_{i} \in$ $\mathcal{L}_{0}^{+}(E, \mathbb{R}), i=1,2, \ldots, n$, and let $\prod_{i=1}^{n} f_{i}{ }^{1 / p_{i}}, \prod_{i=1}^{n} f_{i}^{1 / n} \in \mathcal{L}_{0}^{+}(E, \mathbb{R})$. If $A \in$ $\mathcal{J}(\mathcal{L}(E, \mathbb{R}), \mathbb{R})$, then the following series of inequalities hold:

$$
\begin{aligned}
& n \max _{1 \leq i \leq n}\left\{\frac{1}{p_{i}}\right\}\left[\prod_{i=1}^{n} A^{\frac{1}{p_{i}}}\left(f_{i}\right)-\prod_{i=1}^{n} A^{\frac{1}{p_{i}}-\frac{1}{n}}\left(f_{i}\right) \cdot A\left(\prod_{i=1}^{n} f_{i}^{\frac{1}{n}}\right)\right] \\
& \geq \prod_{i=1}^{n} A^{\frac{1}{p_{i}}}\left(f_{i}\right)-A\left(\prod_{i=1}^{n} f_{i}^{\frac{1}{p_{i}}}\right) \\
& \geq n \min _{1 \leq i \leq n}\left\{\frac{1}{p_{i}}\right\}\left[\prod_{i=1}^{n} A^{\frac{1}{p_{i}}}\left(f_{i}\right)-\prod_{i=1}^{n} A^{\frac{1}{p_{i}}-\frac{1}{n}}\left(f_{i}\right) \cdot A\left(\prod_{i=1}^{n} f_{i}^{\frac{1}{n}}\right)\right] .
\end{aligned}
$$

Proof. The proof is a direct consequence of relation (37). Namely, under notations as in Remark 9 , if we consider $n$-tuple $\mathbf{x}=\left(x_{1}, x_{2}, \ldots, x_{n}\right)$, where 
$x_{i}=\left[f_{i} / A\left(f_{i}\right)\right]^{\frac{1}{p_{i}}}, i=1,2, \ldots, n$, the expressions in (37), that represent the difference between arithmetic and geometric mean, become

$$
\begin{aligned}
M_{1}\left(\mathbf{x}^{\mathbf{p}}, \mathbf{p}^{-1}\right)-M_{0}\left(\mathbf{x}^{\mathbf{p}}, \mathbf{p}^{-1}\right) & =\sum_{i=1}^{n} \frac{f_{i}}{p_{i} A\left(f_{i}\right)}-\prod_{i=1}^{n} \frac{f_{i}^{\frac{1}{p_{i}}}}{A^{\frac{1}{p_{i}}}\left(f_{i}\right)}, \\
A_{n}\left(\mathbf{x}^{\mathbf{p}}\right)-G_{n}\left(\mathbf{x}^{\mathbf{p}}\right) & =\frac{1}{n} \sum_{i=1}^{n} \frac{f_{i}}{A\left(f_{i}\right)}-\prod_{i=1}^{n} \frac{f_{i}^{\frac{1}{n}}}{A^{\frac{1}{n}}\left(f_{i}\right)} .
\end{aligned}
$$

Now, if we apply isotonic functional $A \in \mathcal{J}(\mathcal{L}(E, \mathbb{R}), \mathbb{R})$ on above expressions, and use its linearity property, we get

$$
\begin{aligned}
A\left[M_{1}\left(\mathbf{x}^{\mathbf{p}}, \mathbf{p}^{-1}\right)-M_{0}\left(\mathbf{x}^{\mathbf{p}}, \mathbf{p}^{-1}\right)\right] & =\sum_{i=1}^{n} \frac{A\left(f_{i}\right)}{p_{i} A\left(f_{i}\right)}-\frac{A\left(\prod_{i=1}^{n} f_{i}^{\frac{1}{p_{i}}}\right)}{\prod_{i=1}^{n} A^{\frac{1}{p_{i}}}\left(f_{i}\right)} \\
& =1-\frac{A\left(\prod_{i=1}^{n} f_{i}^{\frac{1}{p_{i}}}\right)}{\prod_{i=1}^{n} A^{\frac{1}{p_{i}}}\left(f_{i}\right)},
\end{aligned}
$$

and

$$
\begin{aligned}
A\left[A_{n}\left(\mathbf{x}^{\mathbf{p}}\right)-G_{n}\left(\mathbf{x}^{\mathbf{p}}\right)\right] & =\frac{1}{n} \sum_{i=1}^{n} \frac{A\left(f_{i}\right)}{A\left(f_{i}\right)}-\frac{A\left(\prod_{i=1}^{n} f_{i}^{\frac{1}{n}}\right)}{\prod_{i=1}^{n} A^{\frac{1}{n}}\left(f_{i}\right)} \\
& =1-\frac{A\left(\prod_{i=1}^{n} f_{i}^{\frac{1}{n}}\right)}{\prod_{i=1}^{n} A^{\frac{1}{n}}\left(f_{i}\right)} .
\end{aligned}
$$

However, by application of functional $A \in \mathcal{J}(\mathcal{L}(E, \mathbb{R}), \mathbb{R})$ on the series of inequalities in (37), the signs of inequalities do not change, since $A$ is linear and isotonic. Thus, the result follows easily after reducing previously derived expressions.

Remark 10. Clearly, the first sign of inequality in (40) yields converse of Hölder's inequality, while the second one yields appropriate refinement. Some related results, for $n=2$, were recently considered in paper [22].

Of course, we can also generate extensions of Hölder's inequality in some other forms. Now we give refinement and converse od Hölder's inequality in quotient form, deduced from relation (36). 
Theorem 4. Let $p_{i}>1, i=1,2, \ldots, n$, be conjugate exponents, let $f_{i} \in$ $\mathcal{L}_{0}^{+}(E, \mathbb{R}), i=1,2, \ldots, n$, and let $\prod_{i=1}^{n} f_{i}{ }^{1 / p_{i}} \in \mathcal{L}_{0}^{+}(E, \mathbb{R})$. Then,

$$
\begin{aligned}
& {\left[\frac{n^{n}}{\prod_{i=1}^{n} A\left(f_{i}\right)}\right]^{\min _{1 \leq i \leq n}\left\{\frac{1}{p_{i}}\right\}} A\left(\left[\sum_{i=1}^{n} \frac{f_{i}}{p_{i} A\left(f_{i}\right)}\right]\left[\frac{\prod_{i=1}^{n} f_{i}^{\frac{1}{n}}}{\sum_{i=1}^{n} \frac{f_{i}}{A\left(f_{i}\right)}}\right]^{n \min _{1 \leq i \leq n}\left\{\frac{1}{p_{i}}\right\}}\right)} \\
& \geq \frac{A\left(\prod_{i=1}^{n} f_{i}^{\frac{1}{p_{i}}}\right)}{\prod_{i=1}^{n} A^{\frac{1}{p_{i}}}\left(f_{i}\right)} \geq\left[\frac{n^{n}}{\prod_{i=1}^{n} A\left(f_{i}\right)}\right]^{\max _{1 \leq i \leq n}\left\{\frac{1}{p_{i}}\right\}} A \cdot \\
& \cdot\left(\left[\sum_{i=1}^{n} \frac{f_{i}}{p_{i} A\left(f_{i}\right)}\right]\left[\frac{\prod_{i=1}^{n} f_{i}^{\frac{1}{n}}}{\sum_{i=1}^{n} \frac{f_{i}}{A\left(f_{i}\right)}}\right]^{n \max _{1 \leq i \leq n}\left\{\frac{1}{p_{i}}\right\}}\right),
\end{aligned}
$$

provided that $A \in \mathcal{J}(\mathcal{L}(E, \mathbb{R}), \mathbb{R})$ is such that all the expressions in (41) are well defined.

Proof. We consider relation (36) in the same setting as in Theorem 3. By inverting, (36) can be rewritten in the form

$$
\begin{aligned}
& M_{1}\left(\mathbf{x}^{\mathbf{p}}, \mathbf{p}^{-1}\right)\left[\frac{G_{n}\left(\mathbf{x}^{\mathbf{p}}\right)}{A_{n}\left(\mathbf{x}^{\mathbf{p}}\right)}\right]^{n \min _{1 \leq i \leq n}\left\{\frac{1}{p_{i}}\right\}} \geq M_{0}\left(\mathbf{x}^{\mathbf{p}}, \mathbf{p}^{-1}\right) \\
& \geq M_{1}\left(\mathbf{x}^{\mathbf{p}}, \mathbf{p}^{-1}\right)\left[\frac{G_{n}\left(\mathbf{x}^{\mathbf{p}}\right)}{A_{n}\left(\mathbf{x}^{\mathbf{p}}\right)}\right]^{n \max _{1 \leq i \leq n}\left\{\frac{1}{p_{i}}\right\}} .
\end{aligned}
$$

Now, if we consider $n$-tuple $\mathbf{x}=\left(x_{1}, x_{2}, \ldots, x_{n}\right)$, where $x_{i}=\left[f_{i} / A\left(f_{i}\right)\right]^{\frac{1}{p_{i}}}$, $i=1,2, \ldots, n$, the expressions that represent means in (42) become

$$
\begin{aligned}
& M_{1}\left(\mathbf{x}^{\mathbf{p}}, \mathbf{p}^{-1}\right)=\sum_{i=1}^{n} \frac{f_{i}}{p_{i} A\left(f_{i}\right)}, \quad M_{0}\left(\mathbf{x}^{\mathbf{p}}, \mathbf{p}^{-1}\right)=\prod_{i=1}^{n} \frac{f_{i}^{\frac{1}{p_{i}}}}{A^{\frac{1}{p_{i}}}\left(f_{i}\right)}, \\
& A_{n}\left(\mathbf{x}^{\mathbf{p}}\right)=\frac{1}{n} \sum_{i=1}^{n} \frac{f_{i}}{A\left(f_{i}\right)}, \quad G_{n}\left(\mathbf{x}^{\mathbf{p}}\right)=\prod_{i=1}^{n} \frac{f_{i}^{\frac{1}{n}}}{A^{\frac{1}{n}}\left(f_{i}\right)} .
\end{aligned}
$$

Finally, if we act with functional $A \in \mathcal{J}(\mathcal{L}(E, \mathbb{R}), \mathbb{R})$ on $(42)$ in described setting, after reduction we get (41).

Remark 11. The second sign of inequality in (41), from the left, yields converse of Hölder's inequality in quotient form. Let's explain why the first inequality in (41) gives refinement of Hölder's inequality. Bearing in mind the notations from the proof of Theorem 4, the quotient of the left-hand side and 
the right-hand side in Hölder's inequality (39) is equal to $A\left(M_{0}\left(\mathbf{x}^{\mathbf{p}}, \mathbf{p}^{-1}\right)\right)$. Since $G_{n}\left(\mathbf{x}^{\mathbf{p}}\right) \leq A_{n}\left(\mathbf{x}^{\mathbf{p}}\right)$, by arithmetic-geometric mean inequality, we have

$$
M_{0}\left(\mathbf{x}^{\mathbf{p}}, \mathbf{p}^{-1}\right) \leq M_{1}\left(\mathbf{x}^{\mathbf{p}}, \mathbf{p}^{-1}\right)\left[\frac{G_{n}\left(\mathbf{x}^{\mathbf{p}}\right)}{A_{n}\left(\mathbf{x}^{\mathbf{p}}\right)}\right]^{n \min _{1 \leq i \leq n}\left\{\frac{1}{p_{i}}\right\}} \leq M_{1}\left(\mathbf{x}^{\mathbf{p}}, \mathbf{p}^{-1}\right)
$$

Clearly, action of isotonic functional $A \in \mathcal{J}(\mathcal{L}(E, \mathbb{R}), \mathbb{R})$ on relation (43) preserves the order of inequalities in (43). Thus, the medium expression in (43) yields refinement of Hölder's inequality since

$$
A\left(M_{1}\left(\mathbf{x}^{\mathbf{p}}, \mathbf{p}^{-1}\right)\right)=\sum_{i=1}^{n} \frac{A\left(f_{i}\right)}{p_{i} A\left(f_{i}\right)}=\sum_{i=1}^{n} \frac{1}{p_{i}}=1 .
$$

It is also well known that Hölder's inequality can directly be deduced from Jensen's inequality in the case of two functions (see [17]). That means that Corollary 1 also provide another class of refinements and converses of Hölder's inequality. We conclude this paper with two results of such type.

Theorem 5. Let $1 / r+1 / s=1$, with $r>1$, let $f, g \in \mathcal{L}_{0}^{+}(E, \mathbb{R})$, and let $A \in \mathcal{J}(\mathcal{L}(E, \mathbb{R}), \mathbb{R})$. If the function $f$ attains minimum and maximum value on set $E$, then the following series of inequalities hold:

$$
\begin{aligned}
{\left[\max _{x \in E} f(x)\right]\left[A^{\frac{1}{r}}(1) A^{\frac{1}{s}}\left(\frac{g}{f}\right)-A\left(\left(\frac{g}{f}\right)^{\frac{1}{s}}\right)\right] } \\
\geq A^{\frac{1}{r}}(f) A^{\frac{1}{s}}(g)-A\left(f^{\frac{1}{r}} g^{\frac{1}{s}}\right) \\
\geq\left[\min _{x \in E} f(x)\right]\left[A^{\frac{1}{r}}(1) A^{\frac{1}{s}}\left(\frac{g}{f}\right)-A\left(\left(\frac{g}{f}\right)^{\frac{1}{s}}\right)\right] .
\end{aligned}
$$

Additionally, if $0<r<1$, then the signs of inequality in (44) are reversed.

Proof. We consider relation (13) from Corollary 1 with arguments $f$ and $p$ respectively replaced with $g / f$ and $f$, where $\Phi(x)=-r s x^{1 / s}$. Clearly, $\Phi^{\prime \prime}(x)=$ $x^{1 / s-2}$, so $\Phi$ is convex function if $x>0$. In this setting, Jessen's functional (7) reads

$$
\begin{aligned}
J\left(\Phi, \frac{g}{f}, f ; A\right) & =A\left(f \Phi\left(\frac{g}{f}\right)\right)-A(f) \Phi\left(\frac{A(g)}{A(f)}\right) \\
& =r s\left[A^{1-\frac{1}{s}}(f) A^{\frac{1}{s}}(g)-A\left(f^{1-\frac{1}{s}} g^{\frac{1}{s}}\right)\right] \\
& =r s\left[A^{\frac{1}{r}}(f) A^{\frac{1}{s}}(g)-A\left(f^{\frac{1}{r}} g^{\frac{1}{s}}\right)\right] .
\end{aligned}
$$


Further,

$$
\begin{aligned}
j\left(\Phi, \frac{g}{f} ; A\right) & =A\left(\Phi\left(\frac{g}{f}\right)\right)-A(1) \Phi\left(\frac{A\left(\frac{g}{f}\right)}{A(1)}\right) \\
& =r s\left[A^{1-\frac{1}{s}}(1) A^{\frac{1}{s}}\left(\frac{g}{f}\right)-A\left(\left(\frac{g}{f}\right)^{\frac{1}{s}}\right)\right] \\
& =r s\left[A^{\frac{1}{r}}(1) A^{\frac{1}{s}}\left(\frac{g}{f}\right)-A\left(\left(\frac{g}{f}\right)^{\frac{1}{s}}\right)\right] .
\end{aligned}
$$

So, if we substitute the obtained expressions $J(\Phi, g / f, f ; A)$ and $j(\Phi, g / f ; A)$ in (13), we get (44).

On the other hand, if $0<r<1$, then $r s<0$. Since the expressions $J(\Phi, g / f, f ; A)$ and $j(\Phi, g / f ; A)$ contain factor $r s$, we conclude that the signs of inequality in (44) are reversed in that case.

Theorem 6. Let $1 / r+1 / s=1$, with $r>1$, let $f, g \in \mathcal{L}_{0}^{+}(E, \mathbb{R})$, and let $A \in \mathcal{J}(\mathcal{L}(E, \mathbb{R}), \mathbb{R})$. If the function $f$ attains minimum and maximum value on set $E$, then the following series of inequalities hold:

$$
\begin{aligned}
& {\left[\max _{x \in E} f(x)\right]\left[A^{s-1}(f) A\left(\frac{g}{f}\right)-\left(\frac{A(f)}{A(1)}\right)^{s-1} A^{s}\left(\left(\frac{g}{f}\right)^{\frac{1}{s}}\right)\right]} \\
& \geq\left[A^{\frac{1}{r}}(f) A^{\frac{1}{s}}(g)\right]^{s}-A^{s}\left(f^{\frac{1}{r}} g^{\frac{1}{s}}\right) \\
& \geq\left[\min _{x \in E} f(x)\right]\left[A^{s-1}(f) A\left(\frac{g}{f}\right)-\left(\frac{A(f)}{A(1)}\right)^{s-1} A^{s}\left(\left(\frac{g}{f}\right)^{\frac{1}{s}}\right)\right] .
\end{aligned}
$$

Further, if $0<r<1$, then the signs of inequality in (45) are reversed.

Proof. Similarly as in Theorem 5, we use relation (13) and consider Jessen's functional $J\left(\Phi,(g / f)^{1 / s}, f ; A\right)$, where $\Phi(x)=x^{s} /(s(s-1))$. It is obvious that $\Phi$ is convex for $x>0$ since $\Phi^{\prime \prime}(x)=x^{s-2}$. We have

$$
\begin{aligned}
J\left(\Phi,\left(\frac{g}{f}\right)^{\frac{1}{s}}, f ; A\right) & =A\left(f \Phi\left(\left(\frac{g}{f}\right)^{\frac{1}{s}}\right)\right)-A(f) \Phi\left(\frac{A\left(f^{\frac{1}{r}} g^{\frac{1}{s}}\right)}{A(f)}\right) \\
& =\frac{1}{s(s-1)}\left[A(g)-A^{1-s}(f) A^{s}\left(f^{\frac{1}{r}} g^{\frac{1}{s}}\right)\right],
\end{aligned}
$$


and

$$
\begin{aligned}
j\left(\Phi,\left(\frac{g}{f}\right)^{\frac{1}{s}} ; A\right) & =A\left(\Phi\left(\left(\frac{g}{f}\right)^{\frac{1}{s}}\right)\right)-A(1) \Phi\left(\frac{A\left(\left(\frac{g}{f}\right)^{\frac{1}{s}}\right)}{A(1)}\right) \\
& =\frac{1}{s(s-1)}\left[A\left(\frac{g}{f}\right)-A^{1-s}(1) A^{s}\left(\left(\frac{g}{f}\right)^{\frac{1}{s}}\right)\right] .
\end{aligned}
$$

Hence, if we insert the above expressions in (13) and multiply the obtained series of inequalities with $s(s-1) A^{s-1}(f)$, we get $(45)$.

Finally, if $0<r<1$, then $s(s-1) A^{s-1}(f)<0$, so in that setting we have reversed inequalities in (45). That completes the proof.

Remark 12. Clearly, relations (44) and (45) provide refinements and converses of Hölder's inequality containing two functions. Some related converses of Hölder's inequality were also obtained in paper [7].

Acknowledgments: This research was supported by the Croatian Ministry of Science, Education, and Sports, under Research Grants 036-11708891054 (first author), 083-0000000-3227 (second author), and 117-1170889-0888 (third author).

\section{References}

[1] J.M. Aldaz, A refinement of the inequality between arithmetic and geometric means, J. Math. Inequal. (2) 4, (2008), 473-477.

[2] J.M. Aldaz, A stability version of Hölder's inequality, J. Math. Anal. Appl. (343) 2, (2008), 842-852.

[3] J.M. Aldaz, Self-improvement of the inequality between arithmetic and geometric means, J. Math. Inequal. (3) 2, (2009), 213-216.

[4] J.M. Aldaz, A measure-theoretic version of the Dragomir-Jensen inequality, RGMIA Research Report Collection (14) Art. 6, (2011).

[5] J.M. Aldaz, Concentration of the ratio between the geometric and arithmetic means, J. Theoret. Probab. (to appear), available at the Mathematics ArXiv.

[6] J.M. Aldaz, Comparison of differences between arithmetic and geometric means, Tamkang J. Math. (to appear), available at the Mathematics ArXiv. 
[7] M. Anwar, J. Pečarić, On logarithmic convexity for differences of power means and related results, Math. Inequal. Appl. (12) 1, (2009), 81-90.

[8] J. Barić, M. Matić, J. Pečarić, On the bounds for the normalized Jensen functional and Jensen-Steffensen inequality, Math. Inequal. Appl. (12) 2, (2009), 413-432.

[9] P.R. Beesack, J.E. Pečarić, On Jessen's inequality for convex functions, J. Math. Anal. Appl. (110) 2, (1985), 536-552.

[10] V. Čuljak, B. Ivanković, J. Pečarić, On Jensen-Mcshane's inequality, Period. Math. Hungar. (58) 2 (2009), 139-154.

[11] S.S. Dragomir, C.E.M. Pearce, J.E. Pečarić, On Jessen's and related inequalities for isotonic sublinear functionals, Acta. Sci. Math. (Szeged) (61) 1-4, (1995), 373-382.

[12] S.S. Dragomir, J.E. Pečarić, L.E. Persson, Properties of some functionals related to Jensen's inequality, Acta Math. Hungar. (70) 1-2 (1996), 129143.

[13] S.S. Dragomir, Bounds for the normalised Jensen's functional, Bull. Austral. Math. Soc. (74) 3, (2006), 471-478.

[14] B. Gavrea, J. Jakšetić, J. Pečarić, On a global upper bound for Jensen's inequality, ANZIAM J. (50) 2, (2008), 246-257.

[15] F.V. Husseinov, On Jensen's inequality, Mat. Zametki (41), (1987), 798806 (Russian).

[16] F. Kittaneh, Y. Manasrah, Improved Young and Heinz inequalities for matrices, J. Math. Anal. Appl. (361) 1, (2010), 262-269.

[17] D.S. Mitrinović, J.E. Pečarić, A.M. Fink, Classical and New Inequalities in Analysis, Kluwer Academic Publishers, Dordrecht/Boston/London, 1993.

[18] J.E. Pečarić, P.R. Beesack On Jessen's inequality for convex functions II, J. Math. Anal. Appl. (118) 1, (1986), 125-144.

[19] J.E. Pečarić, On Jessen's inequality for convex functions III, J. Math. Anal. Appl. (156) 1, (1991), 231-239.

[20] J.E. Pečarić, I. Rasa, On Jessen's inequality, Acta. Sci. Math. (Szeged) (56) 3-4, (1992), 305-309. 
[21] J.E. Pečarić, F. Proschan, Y.L. Tong, Convex functions, partial orderings, and statistical applications, Academic Press, Inc, 1992.

[22] J. Pečarić, V. Šimić, A note on the Hölder inequality, J. Inequal. Pure and Appl. Math. 7 (5) art. 176, (2006), 1-3.

[23] I. Rasa, A note on Jessen's inequality, Itin. Sem. Funct. Eq. Approx. Conv. Cluj-Napoca, Univ. "Babes-Bolyai", preprint 6, (1988), 275-280.

[24] S. Simić, On a global upper bound for Jensen inequality, J. Math. Anal. Appl. (343) 1, (2008), 414-419.

Mario Krnić,

Faculty of Electrical Engineering and Computing,

Unska 3, 10000 Zagreb, Croatia

Email: Mario.Krnic@fer.hr

Neda Lovričević,

Faculty of Civil Engineering and Architecture,

Matice hrvatske 15, 21000 Split, Croatia

Email: neda.lovricevic@gradst.hr

Josip Pečarić,

Faculty of Textile Technology,

University of Zagreb,

Pierottijeva 6, 10000 Zagreb, Croatia

Email: pecaric@element.hr 
